attributable to generic drugs, $20.5 \%$ for the elderly families versus $18.7 \%$ for nonelderly families. Access to data on days of therapy, in addition to prescription (Rx) counts and dosage units, would have helped to further clarify this finding. ${ }^{14}$

The economic burden of prescription drugs for nonelderly and elderly families will increase, at least in the short term. Prescription drug spending is projected to grow by at least $13 \%$ and as much as $20 \%$ in $2003 . .^{15-17}$ An upward spiral of economic burden is created by rising prescription drug prices and prescription drug utilization coincident with stagnant personal income. The perceived burden of prescription drug expenditures also will increase with declining household wealth in the United States, which fell in the third quarter of 2002 to its lowest level since $1995 .{ }^{18}$ The burden for elderly families is not distributed evenly, and prescription drug coverage is associated with higher utilization for elderly persons with ostensibly the same health status. For elderly persons with no chronic disease conditions, drug utilization is more than 2 times (112\%) higher for persons with prescription drug coverage than for persons without prescription coverage. The difference in drug utilization for the elderly with and without prescription drug coverage declines steadily with declining health status. For the elderly with 5 or more chronic disease conditions, the difference in prescription drug utilization is just 15\%, 3.7 prescriptions per person per month for the elderly with prescription drug coverage versus 3.2 prescriptions per person per month for the elderly without prescription drug coverage. ${ }^{19}$ About $76 \%$ of Medicare beneficiaries had prescription drug coverage at some point in $1999 .{ }^{20}$

\section{Preventing Medication Errors and Adverse Drug Events}

The House Energy and Commerce Committee on September 25, 2002, approved a bill to create a confidential, voluntary database that health care providers could use to report medical errors. ${ }^{21}$ The legislation would have to be reconciled with a similar bill approved the previous week by the House Ways and Means Committee and a Senate bill, the Patient Safety and Quality Improvement Act. ${ }^{22}$ The House Energy and Commerce Committee bill would allow patient-safety organizations to monitor the database and use the information to develop recommendations on ways to prevent future mistakes. ${ }^{23}$ The legislative proposal received fuel from a government research report that estimated medical errors cause thousands of deaths and injuries and cost $\$ 29$ billion a year.

Yet, there is disagreement about the scope and severity of the threat to patient safety posed by the U.S. health care system. The first Institute of Medicine report on the matter, To Err Is Human, was released in late 1999 and set off a firestorm of debate about the estimated versus true magnitude of the threat to patient safety in the current U.S. health care system. ${ }^{24}$ This IOM report was criticized for overestimating the incidence of preventable deaths due to medical errors and for adding to the miscommunication on the subject by fostering the interchangeable use of "medical error" and "adverse event." ${ }^{25-27}$ A recent study of physicians and nonphysicians of their first-hand experiences with medical errors helped to provide additional perspective on the perceived severity of the threat to patient safety. Parallel surveys of 831 physicians and 1,207 nonphysicians (adults age 18 or older) conducted between April 11 and June 11,2002 , found that $35 \%$ of physicians and $42 \%$ of the public reported personal experience with medical errors in their own or a family member's care. However, neither group viewed medical errors as one of the most important problems in health care in 2002. ${ }^{28}$ These findings may call into question the sense of urgency to stamp out medical errors expressed by many observers, consultants, and national organizations. The findings of these surveys also appear to add support to those who disagree with the reports of widespread medical errors in the U.S. health care system ${ }^{29}$ and to those critical of patchwork methods to improve health system quality. ${ }^{30}$ Reliable measures are necessary to benchmark and assess the value and return on investment from allocation of finite resources to eliminate errors of commission in health care. Some argue that finite resources might be better spent to reduce errors of omission, such as the failure to control hypertension. ${ }^{31}$

While the debate continues regarding the true magnitude of the threat to patient safety posed by the U.S. health care system, evidence is accumulating regarding the disparity between estimates of medical errors and the actual incidence of medical errors and harm. Fundamental to our understanding is the recognition that a medical error (ME) may or may not be associated with an adverse event (AE). ${ }^{32}$ Similarly, a medication error may result in no harm and no adverse drug event (ADE). ${ }^{33}$ The U.S. Pharmacopeia Center for the Advancement of Patient Safety reported in December 2002 that data reported by 368 health care facilities in 2001 showed 2.4\% of hospital medication errors to have resulted in patient injury or death; the incidence of death from medication error was 14 , or 1.3 per 10,000 medication errors. ${ }^{34}$ A study published in September 2002 based upon direct observation, a method more reliable than other methods, found a 19\% error rate in medications (drug MEs) and a $7 \%$ rate of potentially harmful drug errors (ADEs). ${ }^{35}$

Certainly, the first priority is to prevent the ADEs and adverse medical events (AMEs) with the worst outcomes, death or disability. Categorization and differentiation of medical errors and medication errors from ADEs and AMEs and stratification of the events by level of severity of harm ${ }^{36}$ will permit the focus necessary to allocate the resources to prevent them. It is now well accepted that the preferred method to improve identification and prevention of errors and adverse outcomes from errors involves self-investigation of system causes rather than external review and punishment of organizations and individuals. ${ }^{37-38}$ The method of error reporting is also critical to the identification of true-positive incidences of medical errors, including medication errors. Self-reporting of medication errors may under-report the true incidence by as much as $95 \%,{ }^{39}$ and current methods of collecting information on ADEs may under- 
report the true incidence by as much as $99 \% .{ }^{40}$

In this issue of the Journal, Grissinger, Globus, and Fricker, from the Institute of Safe Medication Practices (ISMP), focus on the patient-practitioner interaction as a primary opportunity to reduce medication errors. ${ }^{41}$ This is also the principal focus of a campaign launched by the U.S. Food and Drug Administration (FDA) in August 2002 that had the theme: "Think through the risks and benefits of medicines." ${ }^{22}$ This consumer-oriented education campaign urged patients to ask questions of their physicians and pharmacists and to become more active in the process of assessing the benefits and risk of prescription drugs: "before using any medicine-as with many things that you do every day-you should think through the benefits and the risks in order to make the best choice for you." Managed care pharmacy can effectively use the same metaphor: when driving a car, you wear your seat belt; when taking medications, you talk to your pharmacist and physician.

Managed care pharmacy can also have a measurable effect on the medication errors and ADEs through reliance on the principles of continuous quality improvement, a fundamental, core area of the Academy of Managed Care Pharmacy's "Pharmacy's Framework for Drug Therapy Management in the 21st Century." The framework's self-assessment tool contains specific tasks and components within key functional areas that permit individual, organization, and system analysis of opportunities for quality improvement in drug therapy management. Key functional areas in patient safety and reduction of medication errors and ADEs are interspersed throughout the framework, with emphasis on patient-practitioner interaction in interpersonal communication (area 1.1), patient education (1.3, 4.4, 5.3, and 6.3), patient and worker safety (1.4), drug selection (3.2), coordination of care (3.5), etc. ${ }^{43}$ Many of the solutions necessary to prevent and reduce medical errors and medication errors will involve changes in processes and systems that "make it easy to do it right." ${ }^{44}$

\section{Quality of Health Economic Studies (OHES) - Tool or Mask?}

Managed care pharmacists face a mountain of data when making decisions about the relative value of alternate drug therapies in individual patients and in the selection of preferred agents in prescription drug formularies. The objective is to apply rules of evidence-based medicine to derive the information that will be important to develop and refine clinical practice guidelines (CPGs) and clinical practice models (CPMs) that will make it possible to achieve effective disease management. This paradigm might be made more clear by thinking of this continuum in terms of structure, process, and outcome in which the "structure" derives from evidence-based medicine, the "process" from application of CPGs and CPMs, and the outcome as successful attainment of disease management.

The U.S. National Library of Medicine reported an average 10,000 new lines (articles) referenced in MEDLINE each week at year-end 2001. ${ }^{45}$ The amount of data and information in the medical literature is growing further and is now quite easily overwhelming, setting aside the additional data and information disseminated in the lay press and on the Internet. It is now more important than ever to find tools to help filter and interpret enormous amounts of data and thousands of medical literature references. The Quality of Health Economics (QHES) instrument may be such a tool. On the other hand, this tool, as any tool, can be misapplied. In addition to possible inherent flaws in the instrument, some of which will only be discovered upon repeated use and scrutiny of the results, users of the QHES have the potential to distort the findings of the studies that they are measuring.

Ofman, Sullivan, Neumann, et al. in this issue of the Journal, take the bold step of introducing a new instrument, the QHES. ${ }^{46}$ The true value of this instrument and method will not be determined immediately, and readers have reason to be critical. Shaya and Lyles in an accompanying editorial suggest that managed care pharmacy should evaluate critically this new instrument and method. ${ }^{47}$ Motheral argues for caution and even rejection of the instrument and method. ${ }^{48}$ Science advances through scholarly debate. By articulating and applying the QHES, Ofman et al. permit others to critique the instrument and method and to propose changes that will enhance value by increasing its validity, reliability, and usefulness. We are certainly in need of better tools to evaluate published data.

The QHES has value, perhaps not so much for its final numeric "score," but in its qualitative analysis of the results of assessment of individual items in the 16-item instrument. Some researchers will no doubt want to change the weight of individual items to improve the utility of the QHES in application to particular uses. The architects of the QHES will need to explain for other researchers that the 3 compound items (numbers 5, 8 and 11) in the QHES require affirmation of both questions. Item number 5, "Was uncertainty handled by: 1) statistical analysis to address random events; 2) sensitivity analysis to cover a range of assumptions?" could have one "yes" and one "no" answer, yet the weight for the item is "9." Item number 8 asks 2 questions and has a weight of "7": "Did the analytic horizon allow time for all relevant and important outcomes?" "Were benefits and costs that went beyond 1 year discounted (3\% to $5 \%)$ and justification given for the discount rate?" Item no. 11 also has 2 questions, with one score of "7": "Were the health outcome measures/scales valid and reliable? If previously tested valid and reliable measures were not available, was justification given for the measures/scales used?" At the least, the QHES instrument and method present us with a useful platform for scholarly debate on attempts to bring more science to pharmacoeconomics, a relatively young discipline still in search of credibility in the scientific community.

\section{Antihypertensive Drug Effects on Renal Function and Myocardial Infarction and Implications of the ALLHAT Study Results}

In managed care we must study the effects of drugs outside the 